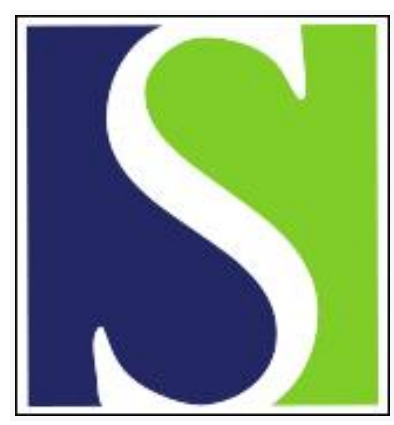

Scand J Work Environ Health 1991;17(2):110-116

https://doi.org/10.5271/sjweh.1729

Issue date: Apr 1991

Evaluation of the physical work load of bricklayers in the steel industry.

by Malchaire JB, Rezk-Kallah B

Affiliation: Catholic University of Louvain, Hygiene and Work Physiology Unit, Brussels, Belgium.

This article in PubMed: www.ncbi.nlm.nih.gov/pubmed/1828613

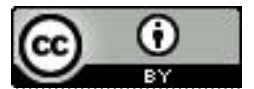




\title{
Evaluation of the physical work load of bricklayers in the steel industry
}

\author{
by Jacques B Malchaire, PhD, ${ }^{1}$ Bagdad Rezk-Kallah, $\mathrm{MD}^{2}$
}

\begin{abstract}
MALCHAIRE JB, REZK-KALLAH B. Evaluation of the physical work load of bricklayers in the steel industry. Scand J Work Environ Health 1991;17:110-6. A study was made of work conditions suspected of being responsible for a greater prevalence of musculoskeletal disorders among 33 bricklayers. A standardized questionnaire was used to confirm a high 12-month prevalence of $73 \%$ for the low back and about $20 \%$ for the upper limbs. Physical work load was studied with the use of heart rate recordings and posture analyses. According to the heart rate data, the work load was "moderately heavy" $(300 \mathrm{~W})$. A diagnostic analysis identified work postures that could be considered as imposing a heavy strain on the musculoskeletal system. The risk associated with these postures was investigated with biomechanical models. This analysis procedure made possible elaborate ergonomic solutions that reduced the work load in general and the biomechanical constraints in particular.
\end{abstract}

Key terms: biomechanics, musculoskeletal disorders, repetitive strain injuries.

In 1988 a study of the incidence of musculoskeletal disorders in a steel factory pointed to a group of bricklayers as one of the most affected groups. A total of 29 disorders, mainly affecting the lower back, had been registered for 33 bricklayers. An analysis of the work conditions that led to these disorders showed that manipulations of heavy objects, especially concrete plates, constituted the riskiest operations.

Consequently, the factory management launched a study to collect the worker's musculoskeletal complaints systematically and to quantify the work load in order to evaluate the causative factors and find ergonomic solutions.

\section{Subjects and methods}

\section{Studied population}

Table 1 shows the means, standard deviations, and ranges of variation of the age, weight, height, and years of experience of the 33 bricklayers. It can be concluded that this was a heterogenous group including subjects varying from definite obesity to marked slimness, some aged, others with few years of experience as a bricklayer.

\section{Description of the bricklayers' work conditions}

The bricklayers' work consisted of overhauling the tundishes. These are large concrete containers serving as temporary stocks between the ladles from the converter and the mold of the continuous casting. After 250 to

\footnotetext{
1 Catholic University of Louvain, Hygiene and Work Physiology Unit, Brussels, Belgium.

2 Oran University, Algeria.
}

Reprint requests to: Professor JB Malchaire, Unité Hygiène et Physiologie du Travail, Clos Chapelle-aux-Champs, 3038, B-1200 Bruxelles, Belgium.
500 ladles, the basic refractory layer of the tundish must be completely stripped off and rebuilt; this extremely hard operation has been transferred to an external specialized firm. In between the rebuildings, after every four or five ladles, the internal coating of the tundish has to be renewed. In this process, the tundish is moved to a brick-laying platform, after being cooled for several hours with a large fan. The operation consists of stripping the inner walls of the tundish by means of a scrapper to expose the underlying concrete surface. The resulting debris is then shoveled away. The bricklayer proceeds to destroy and renew certain parts at the bottom of the tundish, such as the "seatbricks" and the outlets. The demolition is realized with a heavy hammer and a pneumatic hammer. Then new elements are adjusted and cemented into place. This operation is carried out while the worker crouches or leans down to the bottom of the narrow tundish.

The next step is preparing, and then spraying, a mixture of cement constituting the renewed inner coating. The spraying of this mixture is accomplished with the worker inside the tundish carrying the heavy supply pipe full of mixture and its spraying nozzle. An assistant worker helps carry the pipe from the outside of the tundish, a few meters behind the nozzle. Afterwards, the positioning and cementing of the internal concrete barriers take place. These barriers help to regulate the flow of molten metal. The work ends with

Table 1. Characteristics of the group of 33 bricklayers.

\begin{tabular}{lrrc}
\hline Characteristic & Mean & SD & Range \\
\hline Age (years) & 35.5 & 8.5 & $25-48$ \\
Weight $(\mathrm{kg})$ & 82.0 & 12.2 & $55-111$ \\
Height $(\mathrm{cm})$ & 170.5 & 7.4 & $157-184$ \\
Years of experience & 5.2 & 3.6 & $1-12$ \\
\hline
\end{tabular}


complementary operations such as the adjustment of the outlets.

In addition to these main operations, the bricklayers must perform some auxiliary tasks, such as emptying and cooling the tundish, transferring it to the platform, transporting different materials, cleaning the surroundings, etc. This sequence of operations is carried out by two workers, one working mainly inside the tundish and the other on the platform helping the one in the tundish by bringing different tools and materials and supporting the projection pipe. Both workers, however, participate in the scrubbing. Their roles are not fixed, and they can alternate between tasks. A total of 33 bricklayers, divided into four teams, perform these operations on a continuous schedule. The shifts begin at 0600,1400 , and 2200 with a cycle duration of $28 \mathrm{~d}$. On the average, 12 tundishes are repaired daily by three teams of bricklayers.

This study seemed particularly justified, as a reorganization project had been planned by the company. The reorganization called for the work to be performed by one team of bricklayers working from 0700 to 1600 . It would be limited to the current main operations of the bricklayers, while the auxiliary tasks would be done by other shift workers. In this context, the study also aimed at determining the work load that would result from this reorganization.

\section{Medical inquiry}

A standard questionnaire, developed in the Scandinavian countries, was used to collect the history of musculoskeletal disorders during the last 12 months. It gave a general overview of possible musculoskeletal complaints at different anatomic regions and made possible a more-detailed investigation for the most affected parts of the body (1). The questionnaire was filled out by the occupational physician on the basis of the information given by the worker during a private interview.

\section{Job description}

We made a preliminary descriptive study of the job by direct observation and free interviews of the workers in order to gain an understanding of the work procedures, to determine the work cycles, and to try to identify the most strenuous phases of the work.

\section{Heart rate recordings}

Heart rate was recorded with a cardiofrequency meter (Sport Tester ${ }^{\circledR}$ PE 3000) carried by the worker for about 4-h periods. The workers were asked to record their activities periodically while their heart rate was recorded. The results were analyzed by computer in terms of heart rate tracing as a function of time, average heart rate, absolute and relative cardiac costs, and the average equivalent metabolic rate. The absolute cardiac cost was computed as the average heart rate minus the heart rate at rest, while the relative cardiac cost has been defined as absolute cardiac cost divided by the difference between the maximum heart rate predicted from the age and the heart rate at rest. The equivalent metabolic rate was estimated from the average heart rate for an estimation of the average work load in an absolute value, independently of the individual characteristics (2). This equivalent metabolic rate was defined as being the metabolic rate which, on a cycle ergometer, would have been associated with a heart rate equal to the average heart rate observed during the actual work conditions. It was deduced from the heart rate on the basis of the relationship between heart rate and metabolism predicted from the individual characteristics. This relationship has been derived from the resting and maximal values of the heart rate and metabolism. The heart rate surpassed during $99 \%$ of the work period was adopted as the heart rate at rest (2). The resting metabolic rate was considered equal to $105 \mathrm{~W}$, the subject being seated in the conditions that resulted in the recorded resting heart rate. The maximum heart rate $\left(\mathrm{HR}_{\max }\right)$ was evaluated as follows according to the Chaffin expression (3): $\mathrm{HR}_{\max }=190-0.62$ (age - 25). The maximum metabolic rate $\left(\mathrm{M}_{\max }\right)$ was predicted (in watts) as a function of the age and weight of the subject by means of the following expression (4): $M_{\max }=(17.2-0.123$ age) weight.

\section{Posture}

The activities recognized during the preliminary survey as possibly leading to musculoskeletal problems were systematically recorded with a portable video camera. The number of recordings for each of these activities varied from 5 to 15 according to the estimated risk and the complexity of the operations. The filming angle was chosen so as to visualize clearly the whole body and, whenever possible, the profile of the dominant side. Afterwards, the recordings were analyzed in the laboratory by means of a personal computer. The program used was analogous to that developed previously concerning the analysis of work metabolism (5). Starting from a menu, we were able to classify the position of each body segment into a predetermined standard postural category. By analogy with the OWAS (Ovako working posture analysis system) method $(6,7)$, only the main classes of postures were retained, and a more-detailed analysis was carried out for extreme positions. Thus three classes were chosen for the lower limbs (crouching, kneeling, and standing), the back (leaning $<30^{\circ}$, from $30^{\circ}$ to $90^{\circ}$, and $>90^{\circ}$ ), and for the upper limbs (bent $<45^{\circ}, 45^{\circ}$ to $90^{\circ}$, and $>90^{\circ}$ as measured from the vertical plane, the position of the most unfavorable limb being retained). Moreover, three classes of effort were recognized, namely, light $(<3 \mathrm{~kg}$ ), medium ( 3 to $10 \mathrm{~kg}$ ), and heavy $(>10 \mathrm{~kg})$. The classes adopted for the back 
and upper limbs corresponded to positions generally considered as favorable, medium, or unfavorable (8).

Starting from these data, we classified the strain of the global posture for each activity as light, medium, or heavy. Table 2 shows the classification system adopted for medium effort. For light or heavy efforts, the adopted class was respectively one class lower or higher than the one indicated in the table. The lifting of a heavy load while standing with the upper limbs along the body was classified as medium, as was the

Table 2. Classification system adopted for postural strain as a function of the postures of the back, the arms, and the lower legs - medium effort.

\begin{tabular}{|c|c|c|c|}
\hline & Arm $<45^{\circ}$ & Arm $45-90^{\circ}$ & Arm $>90^{\circ}$ \\
\hline $\begin{array}{l}\text { Back }<30^{\circ} \\
\text { Back } 30-90^{\circ} \\
\text { Back }>90^{\circ}\end{array}$ & $\begin{array}{l}\text { Light } \\
\text { Light } \\
\text { Medium }\end{array}$ & $\begin{array}{l}\text { Light } \\
\text { Medium } \\
\text { Heavy }\end{array}$ & $\begin{array}{l}\text { Medium } \\
\text { Heavy } \\
\text { Heavy }\end{array}$ \\
\hline $\begin{array}{l}\text { Crouching } \\
\text { or kneeling }\end{array}$ & Light & Medium & Heavy \\
\hline
\end{tabular}

Table 3. Number of workers complaining of musculoskeletal troubles in different anatomic regions among the 33 bricklayers during the last year or the last week and having been off work during the last year.

\begin{tabular}{|c|c|c|c|}
\hline $\begin{array}{l}\text { Anatomic } \\
\text { area }\end{array}$ & $\begin{array}{c}\text { Trouble } \\
\text { during } \\
\text { the last } \\
12 \text { months }\end{array}$ & $\begin{array}{l}\text { Sick leave } \\
\text { during } \\
\text { the last } \\
12 \text { months }\end{array}$ & $\begin{array}{c}\text { Trouble } \\
\text { during } \\
\text { the last } \\
7 \mathrm{~d}\end{array}$ \\
\hline
\end{tabular}

\begin{tabular}{lrrr} 
Neck & 6 & 1 & - \\
Shoulders & 8 & - & 2 \\
Elbows & 3 & - & - \\
Wrists/hands & 7 & - & 1 \\
Upper back & 3 & 10 & 5 \\
Lower back & 24 & - & 1 \\
Hips/thighs & 2 & - & - \\
Knees & 4 & - & - \\
Ankles/feet & 1 & \\
\hline
\end{tabular}

lifting of a light load with the back and arms bent at $90^{\circ}$. As the aim was to reveal, in a semiquantitative manner, the most strenuous phases of the work, this system, compatible with an analysis at real speed, seemed satisfactory. For the most strenuous phases, a model of prediction of compression forces at the level of the L5-S1 intervertebral disc (9) and a method for computing action limit and maximum permissible limit (10) were used to evaluate the biomechanical risks and to optimize the work conditions.

\section{Results}

\section{Medical inquiry}

Table 3 shows the main results from the general questionnaire on musculoskeletal complaints. The frequency of these complaints in the course of the last 12 months was the highest for the lower back $(72.7 \%)$, shoulders $(24.2 \%)$, neck $(18.2 \%)$, and wrist/hand regions $(21.2 \%)$. During the $7 \mathrm{~d}$ preceding the interview, the frequency of these complaints remained high for the lower back $(15.2 \%)$. Backache was responsible for $30.3 \%$ of the sick leave incurred during the last 12 months. In $45.5 \%$ of the cases, medical care was necessary; a single case was hospitalized. There was no turnover of personnel during that period. The statistical analysis (chi square of contingency) did not reveal any significant effect of individual factors such as age, weight, height, or years of experience on the appearance of these complaints.

\section{Heart rate recordings}

Figure 1 illustrates a typical evolution of heart rate as a function of time for a bricklayer at work. Some of the operations described in the Materials and Methods section are represented. Over a period of $240 \mathrm{~min}$, the actual duration of work was about $61 \mathrm{~min}$, another 33 min were linked to auxiliary activities, while a total

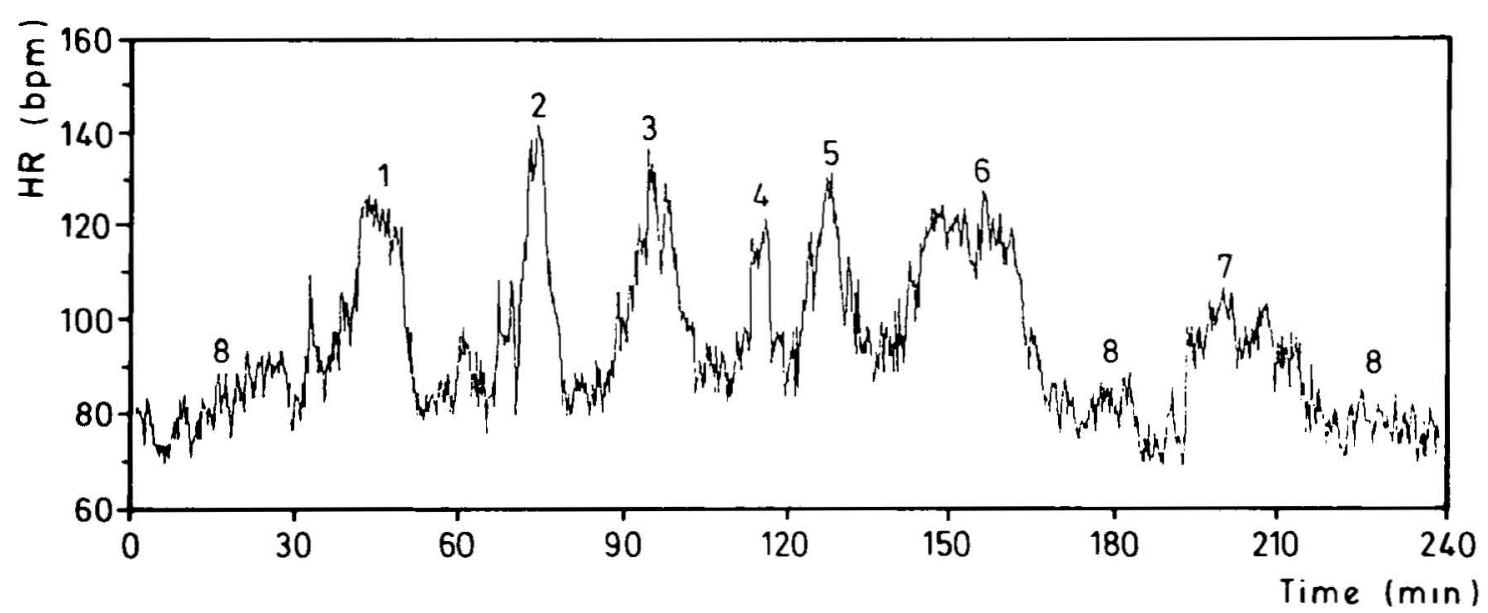

Figure 1. Heart rate profile as a function of time. $(1=$ seat brick demolition, $2=$ cleaning of the tundish, $3=$ setting of the seat brick, $4=$ setting of an outlet, $5=$ adjustment of the outlet, $6=$ cement projection, $7=$ auxiliary activities, $8=$ work break) 
of 145 min (roughly $50 \%$ ) comprised phases of systematic pauses, waiting periods or work breaks.

A total of 27 sequences were recorded for the 33 bricklayers, the mean recorded duration being 226 (SD 25) min with a mean heart rate of 98.5 (SD 11) beats/min. The average heart rate per recording was equal to or higher than 120 beats/min in five cases.

The average equivalent metabolic rate was of the order of $310 \mathrm{~W}$. The physical work load can thus be considered as having been medium heavy and compatible, on the average, with an 8-h period of continuous work. For the three bricklayers presenting the most unfavorable individual characteristics (small, aged, obese), it was higher than $33 \%$ of the maximum work capacity.

\section{Job description}

The heart rate tracings and the direct observations led to a consideration of 11 systematic activities of relatively short duration representing the actual work of the bricklayers (table 4). The other activities, less strenuous, were not analyzed further.

The climatic measurements, carried out at different work sites and particularly inside the tundish, revealed conditions of thermal discomfort [PMV (predicted mean vote) index of 2.13 and PPD (predicted percentage of dissatisfied) index of $80 \%$ ] (11), but with no risk of thermal stress (wet-bulb globe temperature
523) (12). The method proposed by Vogt et al (13) to detect an increase in heart rate of thermal origin did not reveal such an effect in these cases.

Table 4 shows the number of heart rate recordings (no subject had more than two recordings), the overall mean heart rate, absolute and relative cardiac costs, and a qualitative evaluation of the level of energy required for each activity. The most important activities with respect to cardiovascular strain were the cleaning of the tundish, the spraying of the new coating, the replacement of the seat brick, and the lifting of the barriers. The work load could not be estimated for certain activities of short duration or those immediately following a heavy activity.

\section{Posture}

Ninety-three filmed sequences of a total duration of 441 min were studied. Each of the 11 systematic activities was analyzed from 3 to 14 times according to their repetition and degree of complexity. Table 5 gives the mean percentage of time during which the worker adopted the most unfavorable postures and the mean percentage of time for the three levels of global stress for each activity. The major cause of these postures was identified for all cases as being a restricted work space, either in height, while working under the tundish, or in width, while working inside.

Table 4. Number of observations $(\mathrm{N})$, their mean duration, mean heart rate, absolute heart rate, relative heart rate, and the estimated level of metabolism for the main activities.

\begin{tabular}{|c|c|c|c|c|c|c|}
\hline Activity & $\mathrm{N}$ & $\begin{array}{l}\text { Mean } \\
\text { duration }\end{array}$ & $\begin{array}{c}\text { Mean heart } \\
\text { rate } \\
\text { (beats/min) }\end{array}$ & $\begin{array}{c}\text { Absolute } \\
\text { heart rate } \\
\text { (beats/min) }\end{array}$ & $\begin{array}{c}\text { Relative } \\
\text { heart rate } \\
(\%)\end{array}$ & Metabolism \\
\hline $\begin{array}{l}\text { Cement projection } \\
\text { Holding the feeding pipe } \\
\text { Seat brick demolition } \\
\text { Cleaning of the tundish } \\
\text { Adjustment of the outlet } \\
\text { Setting of the seat brick } \\
\text { Setting of accessories } \\
\text { Demolition of the outlet } \\
\text { Setting of the barriers } \\
\text { Setting of a new outlet } \\
\text { Handling of the barriers }\end{array}$ & $\begin{array}{r}14 \\
9 \\
5 \\
6 \\
4 \\
5 \\
2 \\
4 \\
10 \\
6 \\
6\end{array}$ & $\begin{array}{r}14 \min 27 \mathrm{~s} \\
12 \min 43 \mathrm{~s} \\
7 \mathrm{~min} 50 \mathrm{~s} \\
4 \mathrm{~min} 05 \mathrm{~s} \\
3 \mathrm{~min} 40 \mathrm{~s} \\
3 \mathrm{~min} 35 \mathrm{~s} \\
3 \min 35 \mathrm{~s} \\
2 \min 22 \mathrm{~s} \\
2 \min 07 \mathrm{~s} \\
1 \min 34 \mathrm{~s} \\
0 \min 53 \mathrm{~s}\end{array}$ & $\begin{array}{r}117 \\
96 \\
104 \\
118 \\
106 \\
111 \\
\cdots \\
93 \\
\cdots \\
99 \\
111\end{array}$ & $\begin{array}{l}36 \\
20 \\
34 \\
37 \\
34 \\
34 \\
\cdots \\
21 \\
\cdots \\
27 \\
38\end{array}$ & $\begin{array}{l}32 \\
19 \\
30 \\
37 \\
32 \\
32 \\
\cdots \\
18 \\
25 \\
35\end{array}$ & $\begin{array}{l}\text { Heavy } \\
\text { Medium } \\
\text { Heavy } \\
\text { Very heavy } \\
\text { Heavy } \\
\text { Heavy } \\
\text { - } \\
\text { Medium } \\
\text { - } \\
\text { Medium } \\
\text { Heavy }\end{array}$ \\
\hline
\end{tabular}

Table 5. Percentage of time during which the most unfavorable postures were maintained and the level of the resulting strain for the main activities.

\begin{tabular}{|c|c|c|c|c|c|c|}
\hline \multirow{2}{*}{ Activity } & \multirow{2}{*}{$\begin{array}{l}\text { Back } \\
>90^{\circ}\end{array}$} & \multirow{2}{*}{$\begin{array}{l}\text { Arm } \\
>90^{\circ}\end{array}$} & \multirow{2}{*}{$\begin{array}{l}\text { Crouching } \\
\text { or kneeling }\end{array}$} & \multicolumn{3}{|c|}{ Level of strain } \\
\hline & & & & Light & Medium & Heavy \\
\hline Cement projection & 4.7 & 3.9 & 0.8 & 38.6 & 56.2 & 5.2 \\
\hline Holding the feeding pipe & 0.6 & 1.1 & 0.0 & 86.8 & 12.7 & 0.5 \\
\hline Seat brick demolition & 11.4 & 3.0 & 3.0 & 6.1 & 83.0 & 10.9 \\
\hline Cleaning of the tundish & 5.2 & 20.7 & 0.0 & 29.5 & 47.5 & 23.0 \\
\hline Adjustment of the outlet & 2.8 & 34.3 & 9.3 & 24.5 & 40.5 & 35.0 \\
\hline Setting of the seat brick & 33.5 & 10.8 & 21.1 & 30.2 & 38.2 & 31.6 \\
\hline Setting of accessories & 25.1 & 23.8 & 7.9 & 37.0 & 32.5 & 30.5 \\
\hline Demolition of the outlet & 30.3 & 2.1 & 0.0 & 29.5 & 41.2 & 29.3 \\
\hline Setting of the barriers & 5.5 & 9.8 & 0.7 & 46.1 & 46.1 & 7.8 \\
\hline Setting of a new outlet & 14.2 & 13.9 & 7.9 & 56.4 & 28.7 & 14.9 \\
\hline Handling of the barriers & 10.3 & 14.1 & 0.2 & 54.1 & 26.7 & 19.2 \\
\hline
\end{tabular}


A more-detailed analysis of postures and movements was carried out for the phases requiring the most important isometric efforts. The angles of the different body segments were carefully analyzed during the lifting of the 20 to $33 \mathrm{~kg}$ concrete barriers. With the use of the biomechanical model, under the most unfavorable lifting conditions, the compression force at the L5-S1 level has been calculated to be $4000 \mathrm{~N}$ for $50 \%$ of the adult male population (14). This value is higher than the action limit of $3400 \mathrm{~N}$. Therefore the method of analysis of the American National Institute for Occupational Health and Safety (10) was used to allow for the quantification of the actual risk and for the recommendation of required solutions. When a load is picked up at a height of $10 \mathrm{~cm}$ from the ground and at a distance of $40 \mathrm{~cm}$ from the body and lifted to the pelvic level, the action limit is estimated to be $9 \mathrm{~kg}$, while the maximum permissible limit would be $27 \mathrm{~kg}$, that is, less than the actual weight of the heaviest barrier lifted by the bricklayers.

\section{Improvements made in the work conditions}

The relative position of the tundish with respect to the platform appeared to be responsible for the most unfavorable postures adopted by the worker. While the width could not be modified, the level of the platform was adjusted to permit more acceptable conditions above or below the tundish. A height of $180 \mathrm{~cm}$ was adopted to allow an upright posture for the worker during the adjustment of the hoses from underneath the tundish and for the assistant, on the platform, during the lifting of the barriers. Lifting aids were provided to assist the worker in lifting the required materials and tools to the new level.

Nevertheless, the conditions of handling the barriers remained unacceptable for all the workers. The suggested solution was to place the barriers in a pile $60 \mathrm{~cm}$ high over a stand $60 \mathrm{~cm}$ from the platform level. For the most unfavorable condition of lifting (ie, for the last barrier of the pile), the action limit would be $24 \mathrm{~kg}$ and the maximum permissible level $72 \mathrm{~kg}$, as long as the barrier was held vertically and erected against the body before being picked up at both sides. An alternative and more radical solution was the conception of a one-piece barrier (instead of three pieces) weighing $100 \mathrm{~kg}$ and calling for mechanical lifting.

It was possible, through strict cooperation with the technical services of the factory, to find solutions for certain problems, such as the carrying of the spraying nozzle and its feeding pipe. The suggested system consisted of two poles readily raised at both sides of the tundish and connected by a wire easily placed by the workers themselves. A driving wheel made it possible to hang the pipe up, in addition to allowing its free movement along the length of the tundish.

The tools were systematically revised to optimize their mass, form or length, as, for example, scrapers permitting the scraping of the tundish from a stand- ing position outside of the tundish, various types of hammers better adapted to the work to be done, lighter conditioning of certain products, and the like.

\section{Discussion}

A precise determination of the dynamic work load would have required the direct measurement of oxygen consumption. Such a measure would have been very difficult under the practical work conditions of the bricklayers and would have probably resulted, although the views are conflicting in this respect $(15,16)$, in an important perturbation of the mode of work and, therefore, invalidated the results.

The fact that the workers were asked to record their activities periodically does not appear to have influenced their heart rate, as this procedure usually involved simply noting, every 15 or $30 \mathrm{~min}$, a few words describing what was performed during the previous period.

The combined analysis of the heart rate recordings and postures revealed that the heart rate was higher for activities involving both important static postures and great isometric efforts. The static component of heart rate is thus great, and the value of the deduced metabolic rate on the basis of the straight relationship between the heart and metabolic rates can hardly be interpreted in terms of energy expenditure (17). Called equivalent metabolic rate, this value, however, globally qualifies the cardiovascular strain supported by the worker and could serve, together with estimates of the static load, to define work stress.

With the actual work organization, the equivalent metabolism averaged $30 \%$ of the maximal work capacity, and it exceeded the acceptable limit of $33 \%$ (when it was actually referring to energy expenditure) for three workers $(18,19)$.

A reorganization project proposed that the preparation of the tundishes be made by only one team of bricklayers working during the day. It can be foreseen that, in this case, periods of excessive increase in heart rate, such as the ones appearing in figure 1 , would be more frequent and that, without modifications of the work conditions, the resulting work load would be unacceptable for a continuous 8 -h shift. In fact, the real duration of work (during which the mean heart rate equaled 110 beats $/ \mathrm{min}$ ) would increase from 50 to $80 \%$ of the shift time. The mean heart rate over the 8 -h shift would be 106.5 instead of 98.5 beats/min. The equivalent metabolic rate would average $420 \mathrm{~W}$ or $41 \%$ of the maximum work capacity, and would therefore exceed the $33 \%$ limit for the majority of the workers. Such a project, perhaps justified economically, could not be put into effect unless the ergonomic modifications suggested by this study were adopted.

The advantages of heart rate recording at the workplace are, as shown by this study, the gained understanding of the work cycle organization, the ordinal 
classification of different activities from the standpoint of cardiovascular strain, the approximate appreciation of the global work load, and the possibility of predicting the strain inflicted by a different work organization.

The work being essentially static, the analysis of the work load consisted of noting the maintenance time for the different postures and estimating the fraction of time during which the level of stress would be either light, medium, or heavy. The technique used was similar to that of OWAS $(6,7)$ and remains simpler than the methods developed by Corlett et al (20) or Keyserling (8). It allowed for an analysis of video recordings at the actual work speed and for the determination of the most strenuous phases of work, which had to be the object of further analysis. With the use of the biomechanical model, it was shown that, in cases of medium strain, the predicted compression forces at the L5-S1 level were of the order of magnitude of $3400 \mathrm{~N}$. A strain level qualified as being heavy is thus unacceptable for certain workers and requires a moredetailed analysis.

The advantages of a two-step analysis, as used in the present study, are worth emphasizing. Indeed, the principle of a rapid diagnosis followed by detailed analyses of special phases makes the best possible compromise between methods which are either too coarse to arrive at any quantitative conclusion or too elaborated to be compatible with the practical aspects of a field study.

The reproducibility of the results of this method of diagnosis depends, as for all other proposed methods, on the observer's training and on the familiarity with the work conditions. This fact being taken into consideration, the inter- and intraobserver reproducibility was good, as shown in a separate study (21).

\section{Concluding remarks}

The present study shows how a systematic approach can be used routinely to investigate work conditions possibly responsible for an increased prevalence of musculoskeletal disorders, particularly of the lower back. This systematic approach used (i) a standardized questionnaire for the quantification of the one-year incidence of the disorders, (ii) a gross analysis of video recordings of the work situations to classify the different activities on a light, medium and heavy scale concerning the strain of the global posture, based on a rough classification of the back angles, the arm and leg positions, and estimations of the efforts performed, and (iii) more-detailed analysis techniques to determine the risk involved during the "heavy" activities, static biomechanical models being used for fixed postures and the model of the American National Institute for Occupational Safety and Health for lifting operations. In addition, it has been found that heart rate recordings, although irrelevant with respect to the quantifi- cation of the magnitude of isometric efforts, are useful in identifying the most strenuous activities and determining their importance concerning the total cardiovascular strain.

\section{Acknowledgments}

The authors would like to thank the steel factory for permitting this study and the publication of its results, especially Dr Lechien, head of the occupational health department, for his valuable cooperation throughout the study.

The study was supported by a grant from the European Coal and Steel Community.

\section{References}

1. Kuorinka I, Jonsson B, Kilbom A, et al. Standardised Nordic questionnaires for the analysis of musculoskeletal symptoms. Appl Ergon 1987;18:233- 237.

2. Malchaire J, Rogowsky M, Vanderputten M, Wallemacq M. Evaluation de la contrainte de travail par enregistrement continu de la fréquence cardiaque dans une entreprise d'assemblage automobile. Travail Hum 1986; 49:75-87.

3. Chaffin DB. The prediction of physical fatigue during manual labor. J Methods Time Meas 1966;XI- 5: 25-32.

4. Gillet Y. Fréquence cardiaque et consommation d'oxygène au cours d'épreuves d'effort: populations masculine et féminine: observations et prédictions [Mémoire de Licence en Médecine du Travail]. Bruxelles: Université Catholique de Louvain, 1984.

5. Horwat F, Meyer JP, Malchaire J. Validation of a new pocket computer assisted method for metabolic rate estimation in field studies. Ergonomics 1988;31:155164.

6. Karhu O, Kansi P, Kuorinka I. Correcting working postures in industry: a practical method for analysis. Appl Ergon 1977;8:199-206.

7. Karhu O, Harkonen R, Sorvali P, Vepsalainen P. Observing working posture in industry: examples of OWAS application. Appl Ergon 1981;12:13-17.

8. Keyserling WM. Postural analysis of the trunk and shoulders in simulated real time. Ergonomics 1986;29: $569-583$.

9. University of Michigan, Center for Ergonomics. Two dimensional static strength prediction program, Version 4.0c. Ann Arbor, MI: Center for Ergonomics, University of Michigan 1988:52.

10. National Institute for Occupational Safety and Health. Work practices guide for manual lifting. Cincinnati, $\mathrm{OH}$ : US Department of Health and Human Services, 1981:183. (Technical report 81-122.)

11. International Organization for Standardization. Ambiances thermiques modérées: détermination des indices PMV et PPD et spécification des conditions de confort thermique. Geneva: International Organization for Standardization, 1984. (ISO standard $7730-1984$.)

12. International Organization for Standardization. Ambiances chaudes: détermination de l'indice de contrainte thermique WBGT. Geneva: International Organization for Standardization, 1982. (ISO standard 7243 - 1982.)

13. Vogt JJ, Meyer-Schwertz MT, Metz B, Foehr R. Motor, thermal and sensory factors in heart rate variation: a methodology for indirect estimation of intermittent muscular work and environmental heat loads. Ergonomics 1973;16:45-60.

14. Pheasant S. Static anthropometric data. In: Pheasant 
S, ed. Bodyspace: anthropometry, ergonomics and design. London: Taylor and Francis, 1986:67-128.

15. Goldsmith R, O'Brien C, Tan Gle, Smith S, Dixon M. The cost of work on vehicle assembly line. Ergonomics 1978;21:315-323.

16. Malchaire J, Wallemacq $M$, Rogoswsky M, Vanderputten $\mathbf{M}$. Validity of oxygen consumption measurement at the workplace: what are we measuring? Ann Occup Hyg 1984;28:189-193.

17. Nielsen R, Meyer JP. Evaluation of metabolism from heart rate in industrial work. Ergonomics 1987;30: $563-572$.

18. Lehmann G. Praktische Arbeitphysiologie. Stuttgart: Thieme, 1953.

19. Saha PN, Data SR, Banerjee PK, Narayane GG. An ac- ceptable work load for Indian workers. Ergonomics 1979;22:1059-1071.

20. Corlett EN, Madeley SJ, Manenica I. Posture targetting: a technique for recording working postures. Ergonomics 1979;22:357-366.

21. Rezk-Kallah B, Métabolisme et postures de travail, validation d'une méthode d'analyse à partir d'enregistrements vidéo [Mémoire de Licence en Ergonomie]. Bruxelles: Université Catholique de Louvain, 1990.

Received for publication: 17 September 1990 